Meta

Journal des traducteurs

Translators' Journal

\title{
Terminologie de la santé et de la médecine
}

Volume 24, numéro 4, décembre 1979

URI : https://id.erudit.org/iderudit/004513ar

DOI : https://doi.org/10.7202/004513ar

Aller au sommaire du numéro

Éditeur(s)

Les Presses de l'Université de Montréal

ISSN

0026-0452 (imprimé)

1492-1421 (numérique)

Découvrir la revue

Citer cet article

(1979). Terminologie de la santé et de la médecine. Meta, 24(4), 477-480.

https://doi.org/10.7202/004513ar d'utilisation que vous pouvez consulter en ligne.

https://apropos.erudit.org/fr/usagers/politique-dutilisation/ 


\section{TERMINOLOGIE DE LA SANTÉ ET DE LA MÉDECINE}

Annexe à l'arrêté du 7 décembre 1978 de la République française.

Termes nouveaux proposés.

Scanographe (nom masculin) : - Appareil de radiodiagnostic réalisant des coupes d'organes ou de certaines parties du corps et traitant par une calculatrice les données recueillies (en anglais «scanner 》).

Scanographie (nom féminin) : - Méthode de diagnostic utilisant un scanographe.

Liste-annexe à la circulaire du 7 décembre 1978.

Termes dont l'emploi est recommandé ou déconseillé (nouvelles définitions).

$1^{\circ}$ Vocabulaire des toxicomanies

Accoutumance (nom féminin) : - Terme vieilli et ambigu à proscrire.

Assuétude (nom féminin) : - Terme ambigu à proscrire.

Cure de sevrage : - Expression à proscrire.

Dépendance (nom féminin) : - Sujétion à la prise d'une drogue dont la suppression engendre un malaise psychique et/ou des troubles physiques. Désir d'absorber périodiquement ou continuellement une substance pour en retirer du plaisir ou dissiper une sensation de malaise (en anglais : drug dependence).

Dépendance physique : - Etat adaptatif ayant comme conséquence l'apparition de troubles physiques intenses lorsque l'administration de la drogue est suspendue ou après neutralisation de son action par un antagoniste spécifique. Ces troubles constituent un syndrome de sevrage (en anglais : physical dependence).

Dépendance psychique : - État caractérisé par le désir invincible de renouveler la prise d'une drogue sans que s'ensuive nécessairement une tendance à augmenter les doses ou sans qu'apparaisse un syndrome de sevrage en cas de cessation de la prise (en anglais : psychic dependence). 
Drogue (nom féminin) :- Substance naturelle ou synthétique capable d'engendrer une dépendance ; s'emploie également en pharmacologie pour désigner tout produit dont l'administration à l'animal d'expérience provoque une réponse de celui-ci.

Synonyme à déconseiller de médicament. On peut dire aussi drogue simple (en anglais : drug).

Etat de manque : - Terme à remplacer par «état de privation». Synonyme : syndrome de sevrage.

Pharmacodépendance (nom féminin) : - Dépendance à une substance utilisée initialement pour son pouvoir thérapeutique.

Sevrage (nom masculin) : - Suppression du ou des produits auxquels le toxicomane est habitué. Etat du sujet sevré.

Stupéfiant (nom masculin) : - Au sens étymologique se dit d'une substance dont l'administration engendre un état de dépression psychique et/ou physiologique. S'emploie aussi comme synonyme à déconseiller de drogue. Au sens légal, drogue naturelle ou synthétique soumise à une réglementation restrictive touchant sa production, son commerce et son utilisation. En France, produit soumis à la réglementation du tableau $B$ des substances vénéneuses (en anglais : dangerous drug; en américain : narcotic).

Tolérance (nom féminin) : - Propriété que possède l'organisme de supporter sans manifester de réaction l'administration de doses habituellement actives d'une substance déterminée. En matière d'abus de drogue, cette tolérance peut conduire à une augmentation des doses initiales afin de retrouver l'effet recherché (en anglais : tolerance).

Toxicomanie (nom féminin) : - Appétance ou avidité à consommer des substances donnant lieu à dépendance (en anglais : addiction, habit, habituation). $2^{\circ}$ Vocabulaire des greffes

Allogreffe (nom féminin) : - Greffe réalisée entre un donneur et un receveur appartenant à la même espèce mais différant par un ou plusieurs gènes et antigènes d'histocompatibilité.

Allo-immunisation (nom féminin) : - Immunisation d'un individu par un antigène provenant d'un autre individu de la même espèce.

Autogreffe (nom féminin) : - Greffe dans laquelle le greffon est prélevé sur le sujet lui-même.

Greffe allogénique : - Voir allogreffe.

Greffe usogénique : - Voir isogreffe.

Hétérogreffe (nom féminin) :- Greffe réalisée entre un donneur et un receveur appartenant à deux espèces différentes.

Hétérophile (adjectif) : - Se dit d'une même substance à caractère antigénique décelable chez certains êtres vivants appartenant à des espèces différentes. 
Histocompatibilité (nom féminin) : - Ensemble des caractères antigéniques assurant le succès d'une greffe et déterminés par les patrimoines génétiques du donneur et du receveur.

Homogreffe (nom féminin) : - Greffe réalisée entre un donneur et un receveur appartenant à la même espèce et dont les caractères d'histocompatibilité n'ont pas été définis.

Isogreffe (nom féminin) : - Greffe réalisée entre un donneur et un receveur appartenant à la même espèce et possédant les mêmes gènes et antigènes d'histocompatibilité : cette circonstance ne se rencontre que chez les animaux qui appartiennent à la même lignée consanguine et chez l'homme dans le cas de jumeaux univitellins. Employé à tort comme synonyme d'allogreffe.

Iso-immunisation (nom féminin) : - Á éviter. Voir « allo-immunisation ».

Xénogreffe (nom féminin) : - Synonyme peu utilisé d'hétérogreffe : à éviter.

$3^{\circ}$ Vocabulaire de l'andrologie

Anéjaculation (nom féminin) : - Absence d'éjaculation : remplace le terme aspermie inexact.

Asténozoospermie (nom féminin) : - Diminution de motilité des spermatozoïdes.

Azoospermie (nom féminin) : - Absence de spermatozoïdes dans l'éjaculation.

Hypospermie (nom féminin) : — Ejaculation de faible volume.

Hyperspermie (nom féminin) : - Éjaculation de volume important.

Nécrozoospermie (nom féminin) : - Éjaculation de spermatozoïdes morts : remplace le terme nécrospermie.

Oligozoospermie (nom féminin) : - Diminution du nombre de spermatozoïdes : remplace le terme oligospermie inexact.

Polyzoospermie (nom féminin) : - Augmentation du nombre des spermatozoïdes : remplace le terme polyspermie.

Teratozoospermie (nom féminin) : - Augmentation du nombre des spermatozoïdes anormaux.

$4^{\circ}$ Divers

Cardiatomie (nom féminin) : - Incision du cardia. À distinguer de «cardiotomie $»:$ incision $\mathrm{du}$ cour.

Carragénine (nom féminin) : - Substance chimique extraite de certaines algues : orthographe préférable à toute autre.

Effet indésirable (pharmacologie) : - Effet produit par un médicament différent de son effet thérapeutique (en anglais : adverse reaction).

Microrragie (nom féminin) : - Hémorragie minime tachant le linge (en anglais : «spotting 》). 
Néonatologie (nom féminin) : - Étude de la physiologie et de la pathologie du nouveau-né (néo-natalogie doit être proscrit).

Périnatologie (nom féminin) : - Études des phénomènes physiologiques et pathologiques survenant chez la mère et l'enfant immédiatement avant et après l'accouchement (péri-natalogie doit être proscrit).

Xénobiotique (nom masculin, adjectif) : - Substance étrangère à l'organisme vivant. 